\title{
Understanding Scanning Tunneling Microscopy Contrast Mechanisms on Metal Oxides: A Case Study
}

\author{
Harry Mönig, ${ }^{\dagger,+, \S, *}$ Milica Todorović, ${ }^{\perp}$ Mehmet Z. Baykara, ${ }^{\S, \|}$ Todd C. Schwendemann, ${ }^{\S, \#}$ Lucía Rodrigo, $^{\perp}$ \\ Eric I. Altman, ${ }^{\triangle}$ Rubén Pérez, $^{\nabla}$ and Udo D. Schwarz ${ }^{\S}$

\begin{abstract}
${ }^{\dagger}$ Physikalisches Institut, Westfälische Wilhelms-Universität Münster, Wilhelm-Klemm-Strasse 10, 48149 Münster, Germany, ${ }^{\ddagger}$ Center for Nanotechnology (CeNTech), Heisenbergstrasse 11, 48149 Münster, Germany, ${ }^{\S}$ Department of Mechanical Engineering \& Material Science and Center for Research on Interface Structure and Phenomena (CRISP), Yale University, New Haven, Connecticut 06511, United States, ${ }^{\perp}$ Departamento de Física Teórica de la Materia Condensada, Universidad University, 06800 Ankara, Turkey, ${ }^{\# P h y s i c s ~ D e p a r t m e n t, ~ S o u t h e r n ~ C o n n e c t i c u t ~ S t a t e ~ U n i v e r s i t y, ~ N e w ~ H a v e n, ~ C o n n e c t i c u t ~ 06515, ~ U n i t e d ~ S t a t e s, ~}{ }^{\triangle}$ Department of Chemical Engineering and Center for Research on Interface Structure and Phenomena (CRISP), Yale University, New Haven, Connecticut 06511, United States, and ${ }^{\nabla}$ Departamento de Física Teórica de la Materia Condensada and Condensed Matter Physics Center (IFIMAC), Universidad Autónoma de Madrid, 28049 Madrid, Spain
\end{abstract} \\ Autónoma de Madrid, 28049 Madrid, Spain, "Department of Mechanical Engineering and UNAM-Institute of Materials Science and Nanotechnology, Bilkent
}

\begin{abstract}
A comprehensive analysis of contrast formation mechanisms in scanning tunneling microscopy (STM) experiments on a metal oxide surface is presented with the oxygen-induced $(2 \sqrt{2} \times \sqrt{ } 2) R 45^{\circ}$ missing row reconstruction of the $\mathrm{Cu}(100)$ surface as a model system. Density functional theory and electronic transport calculations were combined to simulate the STM imaging behavior of pure and oxygen-contaminated metal tips with structurally and chemically different apexes while systematically varying bias voltage and tip-sample distance. The resulting multipara-

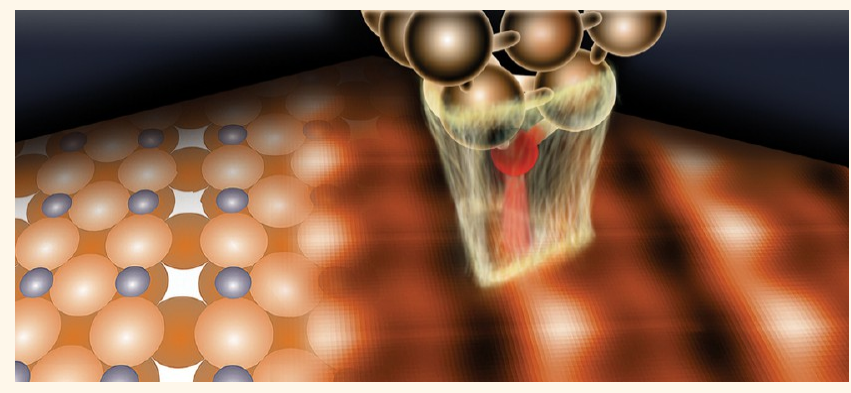
meter database of computed images was used to conduct an extensive comparison with experimental data. Excellent agreement was attained for a large number of cases, suggesting that the assumed model tips reproduce most of the commonly encountered contrast-determining effects. Specifically, we find that depending on the bias voltage polarity, copper-terminated tips allow selective imaging of two structurally distinct surface Cu sites, while oxygenterminated tips show complex contrasts with pronounced asymmetry and tip-sample distance dependence. Considering the structural and chemical stability of the tips reveals that the copper-terminated apexes tend to react with surface oxygen at small tip-sample distances. In contrast, oxygenterminated tips are considerably more stable, allowing exclusive surface oxygen imaging at small tip-sample distances. Our results provide a conclusive understanding of fundamental STM imaging mechanisms, thereby providing guidelines for experimentalists to achieve chemically selective imaging by properly selecting imaging parameters.
\end{abstract}

KEYWORDS: STM contrast $\cdot$ DFT simulation $\cdot$ metal oxide $\cdot$ tip asymmetry $\cdot$ tip chemistry $\cdot$ tip oxidation

M etal oxides form a technologically important class of materials that offers a large variety of bulk and surface properties, enabling applications spanning building materials, machine components, electronic devices, sensors, actuators, and highly efficient catalysts. ${ }^{1}$ In many cases, atomic-scale control of specific structural details is mandatory for the realization of such devices, in particular when interfaces with other materials are formed. ${ }^{2-5}$ For conducting metal oxides, related studies often employ scanning tunneling microscopy (STM) due to the method's ability to deliver images of surfaces with atomic-scale contrasts. ${ }^{6-9}$ The interpretation of STM data, however, is often not straightforward, as the tunneling current represents a convolution of the local electronic properties of both the sample and the tip. The tip is of particular concern, as the chemical identity of its front atom is usually unknown. Moreover, tip apexes with asymmetric shapes can lead to distorted STM images where correlating current maxima with atomic sites can be questionable. Therefore, proper evaluation of experimental data requires comparison with theory.
* Address correspondence to harry.moenig@uni-muenster.de.

Received for review August 30, 2013 and accepted October 10, 2013.

Published online October 10, 2013 $10.1021 / \mathrm{nn} 4045358$

() 2013 American Chemical Society 
Recent years have seen significant progress toward including the probe tip in STM simulations. ${ }^{10-17}$ In the first theoretical studies of STM, featureless metallic tips were considered as suitable probe models. ${ }^{18}$ Since then, it has been shown that the exact geometry and chemistry of the tip termination strongly influence STM contrast formation. ${ }^{11,19}$ For example, different species at the apex can produce varied STM contrasts depending on the orientation of the tip with respect to the surface and the spatial arrangement of the outer shell orbitals involved in tunneling. ${ }^{14,15,20}$ Moreover, since atoms such as nitrogen or oxygen conduct poorly in comparison to metals, current contributions from neighboring metallic tip atoms may become important in the case of $\mathrm{N}$ - or O-terminated tips, which can result in anomalous effects such as contrast inversion. ${ }^{11,10,21}$ Contrast inversion can also occur at varied tip-sample distance due to site-specific variations in the local potential barrier ${ }^{22}$ or multiple scattering current contributions in scans closer to the surface, ${ }^{23}$ further complicating the interpretation of STM images. Finally, tip-surface interactions affect tunneling by deforming the tip and sample to various degrees as a function of both tip-sample distance and lateral probe position; theoretical studies have confirmed the importance of tip relaxation for the correct description of the STM contrast formation. ${ }^{10}$ In a more subtle effect, weak surface interactions or external manipulation can alter the orbital hybridization of the tip-terminating atom and thus modify tunneling properties. ${ }^{12}$ Although the increased focus on the role of the probe in STM imaging has led to remarkable progress in control and functionalization of STM tips by, for example, the attachment of small molecules, ${ }^{13,14}$ a systematic analysis of STM imaging capabilities as a function of all parameters relevant to contrast formation including tip chemistry and structure is still lacking. A complete understanding of all related effects would be of great value, as it is a prerequisite for the ability to take full advantage of the complementary information revealed under different imaging conditions.

By combining high-resolution STM experiments with density functional theory (DFT) simulations and nonequilibrium Green's function (NEGF) calculations on a prototype metal oxide surface, the present study makes significant progress toward this goal. Due to its technological relevance as well as favorable structural properties that aid the unambiguous assignment of lattice sites, the well-known oxygen-induced $(2 \sqrt{ } 2 \times \sqrt{ } 2) R 45^{\circ}$ missing row reconstruction of the $\mathrm{Cu}$ (100) surface ${ }^{24-26}$ has been chosen as a model system. First, we provide an overview of computed STM contrasts as a function of bias voltage, tip-sample distance, and tip chemistry and structure with special consideration of tip asymmetry effects. Next, we analyze the mechanisms that give rise to the observed contrasts, which includes identifying the signatures

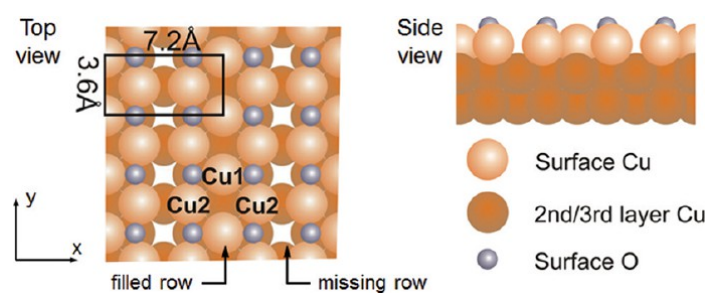

Figure 1. Schematic of the oxygen-induced missing row reconstruction of the $\mathrm{Cu}(100)$ surface, with the field of view reflecting the surface area covered by the simulations presented in upcoming sections. Due to the rotational symmetry of the $\mathrm{Cu}(100)$ surface, the $x$ and $y$ directions may correspond to either the $[010] /[001]$ or the $[001] /[0 \overline{1} 0]$ crystallographic directions of the substrate, respectively. The black rectangle in the top view outlines the size of the unit cell.
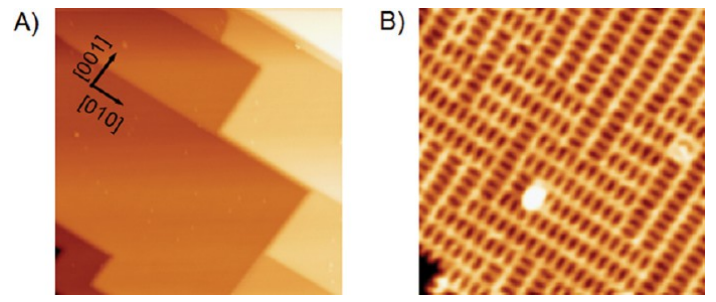

Figure 2. Experimental STM images of the $\mathrm{Cu}(100)-\mathrm{O}$ surface. (A) Large-scale image $(75 \mathrm{~nm} \times 75 \mathrm{~nm})$ revealing the step-edge pinning along the [010] and the [001] directions. (B) High-resolution image $(9 \mathrm{~nm} \times 9 \mathrm{~nm})$ illustrating orthogonal domains also oriented along the [010] and [001] directions; note that domains may be as small as two unit cells. Imaging parameters: $(\mathrm{A})+1.0 \mathrm{~V} / 0.9 \mathrm{nA}$; (B) $-1.1 \mathrm{~V} / 0.4 \mathrm{nA}$.

that oxygen atoms and different copper sites cause in simulated images. The theoretical results provide a solid basis for the subsequent comprehensive comparison with experimental STM data, where we identify and classify the most commonly encountered contrast mechanisms. On the basis of this analysis, we discuss the chemical and structural stability of the model tips and derive general guidelines to understand their imaging characteristics. Most notably, a combination of tip-apex chemistry control with carefully chosen variations in imaging parameters emerges as a promising avenue to enhance the information that scanning tunneling microscopy experiments are able to provide on complex surfaces.

\section{RESULTS}

General Features of the Model Surface. Figure 1 shows the structural model of the $(2 \sqrt{2} \times \sqrt{2}) R 45^{\circ}$ missing row reconstruction $(\mathrm{Cu}(100)-\mathrm{O}$ surface), highlighting its rectangular unit cell as well as the characteristic rows of alternating $\mathrm{O}$ and $\mathrm{Cu}$ atoms $(\mathrm{O}-\mathrm{Cu}-\mathrm{O}$ rows) that are separated either by a row of Cu atoms (labeled as "filled row" in Figure 1) or by a row of vacant sites referred to as "missing row". This arrangement leads to two distinct types of copper atoms: Cu1 sites within filled rows and $\mathrm{Cu} 2$ sites within the $\mathrm{O}-\mathrm{Cu}-\mathrm{O}$ rows. Experimental STM images of the surface are presented in Figure 2, 
with Figure 2A showing the surface's characteristic step-edge pinning along the [010] and the [001] crystallographic directions. ${ }^{25}$ Another feature of this surface oxide layer is the formation of two domain orientations, which nucleate with equal structure and probability along the rotationally symmetric [010] and [001] directions of the $\mathrm{Cu}(100)$ surface. $^{24}$ Typical experimentally observed domain patterns are exemplified by the image in Figure 2B, where both domain orientations show a ladder-type contrast.

The most important advantages of using the $\mathrm{Cu}(100)-\mathrm{O}$ surface as a model system rely on the nearly coplanar sublattices of $\mathrm{Cu}$ and $\mathrm{O}$, which facilitates lattice site identification and allows analysis of the tunneling characteristics of anionic and cationic species. Furthermore, imaging two orthogonal domains in one scan frame enables the investigation of differences in contrast due to the rotational alignment of the tip with respect to the domain orientation.

Choosing Model Tips for Tunneling Current Simulations. In STM experiments, tip-surface contacts during approach or scanning are generally unavoidable. Furthermore, considering that copper is known to easily attach to scanning probe microscopy tip apexes, ${ }^{27-29}$ we expect the tip to be contaminated with surface material. Thus, we considered $\mathrm{Cu}$ and $\mathrm{O}$ as the basic elements for the tip structures used in our simulations instead of tungsten or platinum/iridium, the original tip materials.

For the present study, we constructed a copperbased, atomically sharp STM probe by appending a tetrahedral tip base to a three-layer slab of Cu atoms in a [111] orientation (Figure $3 \mathrm{~A}$ ). This construction mimics the right boundary conditions (in a real tip, the terminating tip atoms protrude out of a large mesoscopic structure) and provides the electronic structure input for the subsequent tunneling current simulations. Please note that the close-packed (111) surface represents the crystallographic plane with the lowest surface energy, which furthermore allows the formation of atomically sharp tips in the [111] direction. Therefore, we expect this tip configuration to be particularly favorable. The tip apex consists of the two lowest layers of the tip, i.e., the terminating atom and a triangular plane of upper $\mathrm{Cu}$ atoms. The terminating atoms were interchanged to feature $\mathrm{Cu}, \mathrm{O}$, and mixed $\mathrm{Cu}-\mathrm{O}$ species as shown in Figure 3, allowing us to simulate STM imaging as a function of tip chemistry. For the tip termination we took into account only the simplest and energetically most stable apex structures with $\mathrm{Cu}$ and $\mathrm{O}$ as the basic elements. While the slab and base of the STM probe are bulk derived, the geometry of the different tip apexes was fully optimized to obtain stable tip structures. Besides the highly symmetric, pure $\mathrm{Cu}$ tip (Figure $3 \mathrm{~B}$ ), we have considered a case where oxygen contamination is achieved by replacing the terminating copper atom by oxygen ("CuO" tip).
A) [111]

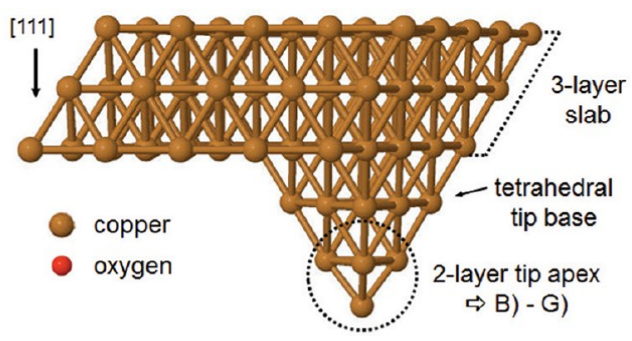

B)

$\mathrm{Cu}$

C)

Cu-rot
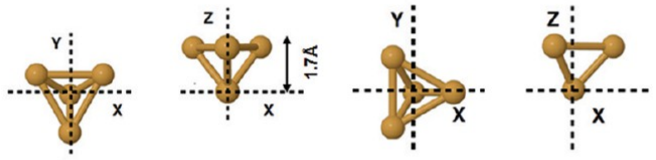

D)

$\mathrm{CuO}$

E) CuO-rot
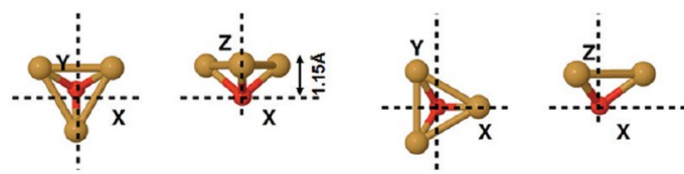

F) $\quad \mathrm{Cu}-\mathrm{CuO}$

G) $\quad \mathrm{Cu}-\mathrm{CuO}-\mathrm{rot}$
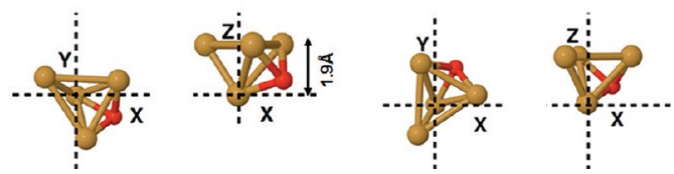

Figure 3. (A) STM tip model constructed by appending the two-layer apex (dashed circle) of panel B to a single-layer tetrahedral base (arrow), which itself is coupled to a [111] oriented trilayer slab. (C-G) Top and side views of alternate tip-apex configurations used in the calculations; these models replace the apex atoms circled in $A$.

Figure 3D demonstrates that the tip-apex geometry remains qualitatively similar, apart from a notable reduction in distance between the lowest atom and the upper Cu layer from $1.7 \AA$ to $1.15 \AA$. We have also introduced a tip where the tetrahedral apex is contaminated by an $\mathrm{O}$ atom attached to one of the tetrahedron's side faces ("Cu-CuO" tip), as seen in Figure 3F.

Our STM simulations go beyond the usual approximation of considering only the lowest apex atom by including the tunneling current flowing between the atoms in the two lowest tip layers and the top layer of the $\mathrm{Cu}(100)-\mathrm{O}$ surface. With this more realistic approach, it is clear that even the simplest, purely metallic Cu tip may break the symmetry of the simulated STM image: Since the alignment of the triangular plane of $\mathrm{Cu}$ atoms above the terminating tip atom represents a particular orientation with respect to the surface's 2-fold symmetry axis, the sensitivity of imaging modes to the tip's rotational alignment ( $z$-axis rotation) needs to be explored. To investigate these tip-asymmetry effects, we have considered an additional case (" $\mathrm{Cu}$ -

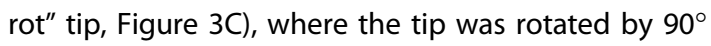
relative to the " $\mathrm{Cu}$ " tip shown in Figure 3B. This rotation check was performed for all chemical terminations of the tip, as illustrated in Figure $3 B-G$, where the 

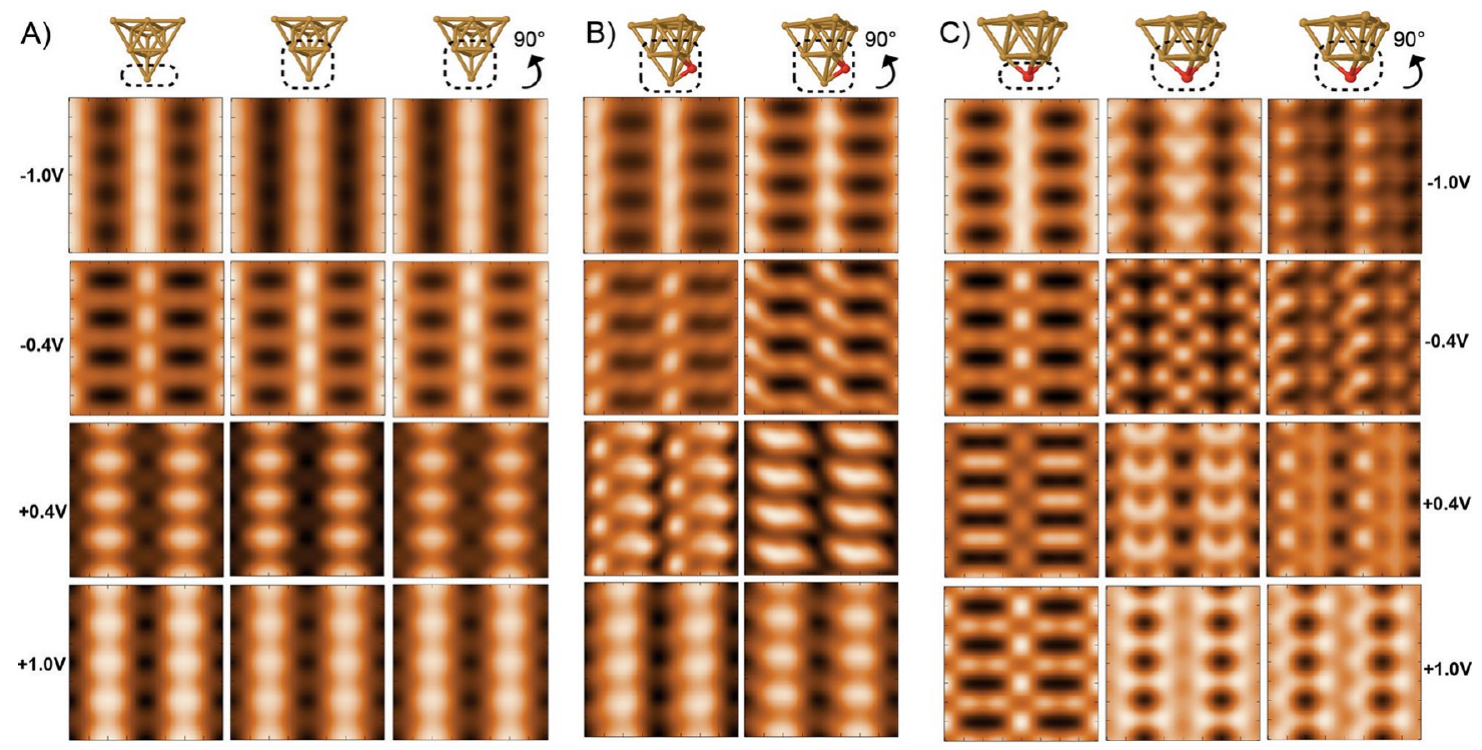

Figure 4. Current maps ( $1.44 \mathrm{~nm} \times 1.44 \mathrm{~nm})$ simulated for the $\mathrm{Cu}(\mathrm{A})$, the $\mathrm{Cu}-\mathrm{CuO}(\mathrm{B})$, and the $\mathrm{CuO}(\mathrm{C})$ tips at different bias voltages and a constant tip-sample distance of $d=5 \AA$. For the calculations, tunneling currents are considered either only from the front tip atom (first columns in $\mathrm{A}$ and $\mathrm{C}$ ) or by considering contributions to the tunneling current from all atoms in the double-layer apex; the tip atoms involved are encircled by dashed lines in the tip models at the top of each column. To study tip-asymmetry effects, the last column of each panel contains simulations where the tips were rotated by $90^{\circ}$, as specified in Figure 3.

"CuO-rot" (Figure 3E) and "Cu-CuO-rot" tips (Figure 3G) have been introduced. For the $\mathrm{Cu}-\mathrm{CuO}$ and $\mathrm{Cu}-\mathrm{CuO}-$ rot tips, the broken symmetry becomes particularly prominent, as the rotation changes the relative orientation of the contaminant $\mathrm{O}$ atom with respect to the filled and missing rows on the surface. Due to the orthogonal domains of the $\mathrm{Cu}(100)-\mathrm{O}$ surface, related differences in contrast are experimentally accessible by recording scan frames covering more than one domain orientation.

Contrast Simulations. The model tips have been used to simulate constant-height STM images as a function of bias voltage with the tip-sample distance fixed at $d=5.0 \AA$; the resulting variety of imaging modes is shown in Figure 4 . The computed $1.44 \mathrm{~nm} \times 1.44 \mathrm{~nm}$ current maps correspond exactly to the section of the $\mathrm{Cu}(100)-O$ model surface displayed in Figure 1, and all voltages are given in terms of sample bias. Therefore, positive bias corresponds to imaging of empty sample states and negative bias to filled states. It should be noted that initially we performed both constant height and constant current simulations, but the resulting image contrasts were qualitatively identical regardless of the specific tip. Consequently, the more efficient constant-height calculations were used for further work. For each tip and bias voltage, separate current maps were produced for the two distinct rotational orientations of the tip relative to the model surface. In addition, we generated current maps based on the contribution exclusively originating from the lowest apex atom for the $\mathrm{Cu}$ and the $\mathrm{CuO}$ tips, as indicated by the dashed circles at the top of each column.

We first note that images generated with the pure Cu tip (Figure 4A) essentially display surface Cu atoms:
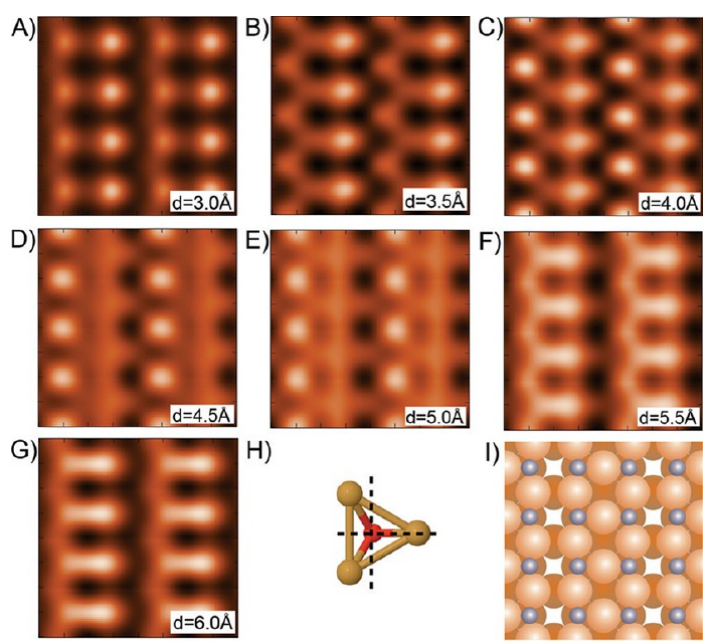

Figure 5. (A-G) Sequence of simulated current maps for the CuO-rot tip at $U_{\text {bias }}=+0.4 \mathrm{~V}$ to illustrate the dependence of the contrast on the tip-sample distance $d$. $(\mathrm{H})$ Schematic top view of the tip apex, highlighting its alignment with respect to the orientation of the surface. (I) Model surface cell used for the simulations.

at negative bias voltages the contrast is dominated by the filled states of $\mathrm{Cu} 1$ atoms and at positive bias voltages by the empty states of Cu2 atoms. Furthermore, our simulations show that the effect of tip rotation is negligible and that the STM contrasts are almost completely dominated by the current passing through the terminating $\mathrm{Cu}$ atom with just a very small background contribution from the upper plane of $\mathrm{Cu}$ atoms. For the side-contaminated $\mathrm{Cu}-\mathrm{CuO}$ tip, the image contrasts in Figure $4 \mathrm{~B}$ at high bias voltages appear very similar to those found for the pure $\mathrm{Cu}$ 
tip. For small bias voltages, however, the image features shift with respect to the underlying surface atoms, which leads to notable contrast changes when the tip is rotated. This effect can be understood by considering the contributions to the tip density of states originating from both the terminating $\mathrm{Cu}$ atom and the contaminant $\mathrm{O}$ atom. For small bias voltages (i.e., close to the Fermi energy) their contributions to the overall density of states of the tip are very similar. This causes the location where the maximum tunneling current flows to move away from the terminating $\mathrm{Cu}$ atom toward the contaminant $\mathrm{O}$ atom. The resulting tip asymmetry effects range from a simple offset of $\sim 1 \AA$ of the current maxima ${ }^{30}$ to wave-like patterns seemingly incompatible with the $\mathrm{Cu}(100)$-O surface symmetry. In contrast, at higher bias voltages (i.e., higher energies) the tip density of states is dominated by the terminating $\mathrm{Cu}$ atom, which minimizes these asymmetry effects.

Compared to the $\mathrm{Cu}$ and the $\mathrm{Cu}-\mathrm{CuO}$ tips, the contrast mechanisms of the $\mathrm{CuO}$ tip are significantly more complex. Current maps simulated by exclusively considering the terminating $\mathrm{O}$ atom (left column in Figure 4C) differ substantially from the maps obtained when including the second layer $\mathrm{Cu}$ atoms, revealing a significant contribution from these $\mathrm{Cu}$ atoms to the overall current for this tip. As a consequence of the triangular geometry of the second layer $\mathrm{Cu}$ atoms, contrast features undergo drastic changes when the tip is rotated by $90^{\circ}$. This is demonstrated by the images in the center and right column of Figure $4 C$, which show significantly different contrasts for the two tip orientations.

To check for possible changes in contrast with tip-sample distance, we computed current maps for distances between $d=3.0 \AA$ and $d=6.0 \AA$ in $0.5 \AA$ increments for all tip configurations and a variety of bias voltages. For the $\mathrm{Cu}$ and $\mathrm{Cu}$-rot configurations, hardly any effect upon changing the distance within this range is observed, while for the $\mathrm{Cu}-\mathrm{CuO}$ and $\mathrm{Cu}-\mathrm{CuO}$-rot tips, a weak distance dependence was found. Unlike these cases, the O-terminated tips (CuO and (uO-rot) show complex and strongly distancedependent image patterns with pronounced asymmetry and the possibility of exclusive oxygen imaging at small tip-sample distances $(d<3.5 \AA$ ), as exemplified by Figure 5 .

The complex imaging properties of the $\mathrm{CuO}$ and CuO-rot tips can be explained by the significant contributions to the tunneling current originating from the second-layer copper atoms. The emergence of this additional current channel is aided by the reduced distance between first- and second-layer atoms $(1.15 \AA$ as opposed to the $1.7 \AA$ displayed by the Cu tip; cf. Figure $3 \mathrm{~B}$ and $\mathrm{D}$ ). Moreover, the tunneling characteristics of the O-terminated tip structure are governed by a distance-dependent interplay of localized $\mathrm{O}$ orbitals and comparatively delocalized $\mathrm{Cu}$ orbitals of both the tip and the sample (see discussion below).

Comparison with Experimental STM Images. For the present work, we evaluated a database of about 100 highquality constant-current STM images recorded on the $\mathrm{Cu}(100)-\mathrm{O}$ surface. All images in the database were first classified into representative contrasts; for each contrast class, we then determined the tip configuration(s) that most likely produced this particular type of contrast by comparison with theory. Once a candidate tip was identified, we performed targeted simulations for selected images using the presumed tip and the experimental bias voltage to calculate a set of images at various tip-sample distances that were then qualitatively compared to the experimental data. A variety of such comparisons is presented in Figures 6-8, where each experimental image is shown with the best match added as both an inset and an enlarged image. The enlarged images are always displayed in the orientation and size defined by the surface model in Figure 1 using tip apexes oriented as shown in Figure 3B-G; dashed lines connect this standardized alignment with the alignment of the inset.

Figure $6 \mathrm{~A}$ shows a zoom of the area in the lower right of Figure $2 \mathrm{~B}$ with two large domains. For both domains a ladder-type contrast is observed at negative bias, which, according to Figure 4, points to a Cu-terminated tip. Therefore, the bright rows can be

A)

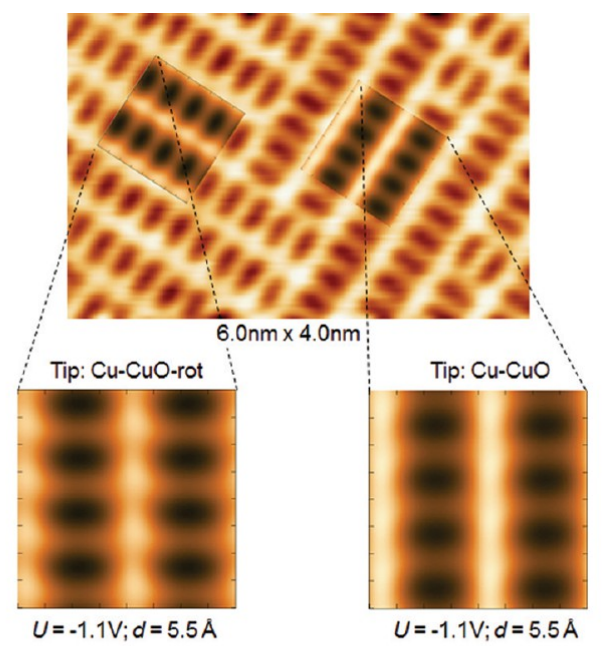

B)

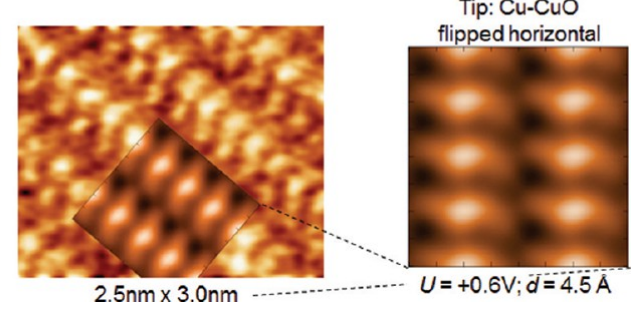

Figure 6. Comparison of experimental and simulated STM contrasts for cases where the analysis suggests $\mathrm{Cu}-\mathrm{CuO}$ and $\mathrm{Cu}-\mathrm{CuO}-$ rot tip configurations. The imaging parameters for the experimental constant current data were $-1.10 \mathrm{~V} / 0.40 \mathrm{nA}(\mathrm{A})$ and $+0.60 \mathrm{~V} / 0.70 \mathrm{nA}(\mathrm{B})$. 
A)

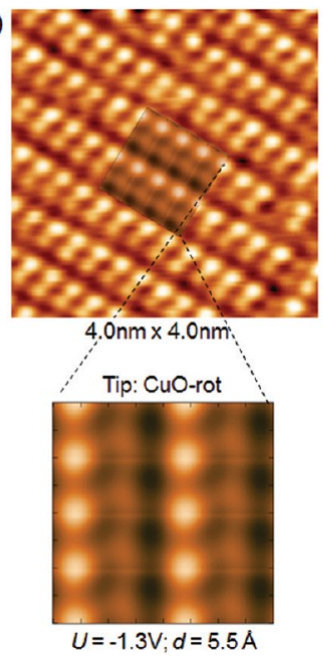

B)

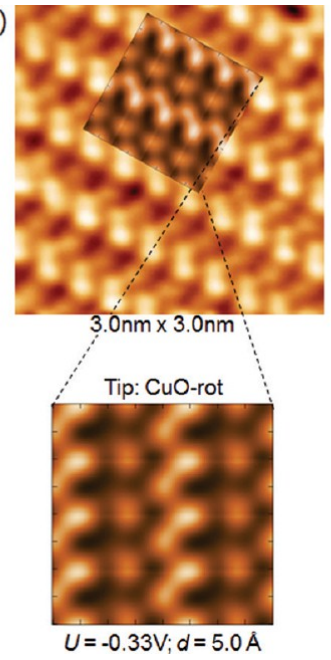

C)

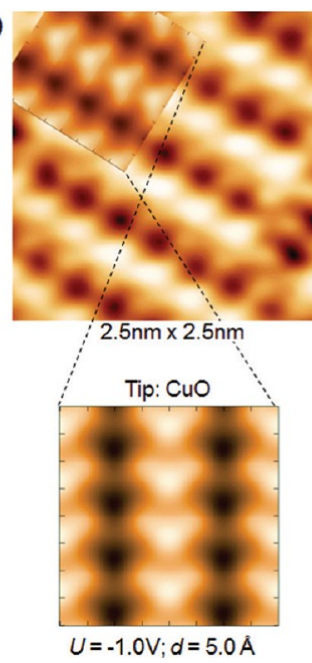

D)

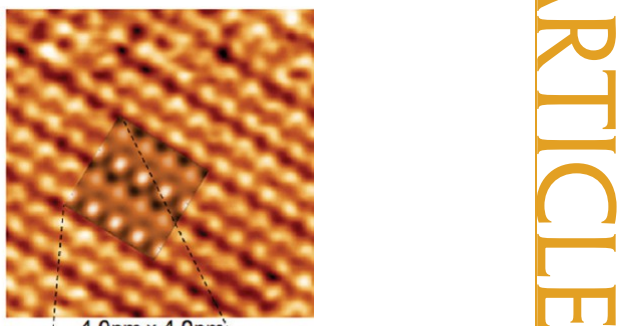

Figure 7. Comparison of experimental and simulated STM contrasts where $\mathrm{CuO}$ and CuO-rot tip configurations have been identified. Imaging parameters for the experimental data were $-1.30 \mathrm{~V} / 0.43 \mathrm{nA}(\mathrm{A}),-0.33 \mathrm{~V} / 0.64 \mathrm{nA}(\mathrm{B}),-1.00 \mathrm{~V} / 0.30 \mathrm{nA}(\mathrm{C})$, and $+0.40 \mathrm{~V} / 0.70 \mathrm{nA}(\mathrm{D})$.

associated with the filled rows, while the Cu2 atoms merge to form slightly darker bridges and appear as the "rungs of the ladder". The best match with theory is found by considering the O-contaminated $\mathrm{Cu}$-CuO tip configurations at a distance of $5.5 \AA$. Since the tip orientation relative to the direction of the $\mathrm{O}-\mathrm{Cu}-\mathrm{O}$ rows affects contrast formation in this case, an identical tip at the same distance but rotated by $90^{\circ}$ has to be assumed for the simulation of the two different domain orientations. Consequently, we considered the $\mathrm{Cu}-\mathrm{CuO}$ for one domain and the $\mathrm{Cu}-\mathrm{CuO}-$ rot tip for the other domain. The result agrees with the experimental contrast, which shows only minor differences between the two domains. The image in Figure 6B shows a wave-like contrast, similar to the simulation shown in Figure $4 \mathrm{~B}$ at $+0.4 \mathrm{~V}$. Therefore, we performed simulations for the $\mathrm{Cu}-\mathrm{CuO}-$ rot and $\mathrm{Cu}-\mathrm{CuO}$ tips at $+0.6 \mathrm{~V}$ and found the best match by assuming the $\mathrm{Cu}-\mathrm{CuO}$ tip at $4.5 \AA$. In this imaging mode the Cu2 atoms appear as joint maxima bridging the missing rows. The maxima asymmetrically fade to the left and the right, leading to the wave-like contrast.

The image in Figure 7A shows a pronounced asymmetry perpendicular to the $\mathrm{O}-\mathrm{Cu}-\mathrm{O}$ rows, suggesting the CuO-rot tip as a likely candidate. This is confirmed by a good match at $5.5 \AA$, which nicely reproduces the experimental contrast at the corresponding bias voltage. In this case, the Cu1 sites are imaged as minima in the dark trenches, whereas the Cu2 atoms located to the right of the Cu1 atoms (Cu2-right) are imaged significantly brighter than the Cu2 atoms left of the Cu1 sites (Cu2-left). This contrast signature is a direct result of significant tunneling current via the upper plane of $\mathrm{Cu}$ atoms of the CuO-rot tip. For example, when the terminating $\mathrm{O}$ atom of the tip is located above Cu2-right sites, the two left-side $\mathrm{Cu}$ atoms in the second layer of the tip are closer to the two neighboring Cu1 atoms of the surface, leading to an increased tunneling current. In contrast, when the terminating $\mathrm{O}$ atom is located above Cu2-left sites, the same two $\mathrm{Cu}$ atoms of the tip are located above the missing row, which decreases the tunneling current, thus leading to a darker contrast of the $\mathrm{Cu}-2$ left atoms. In a similar manner the experimentally observed contrasts in Figure 7B-D can be understood. In Figure 7B Cu2right and Cu1 atoms merge into a zigzag formation, while the Cu2-left sites appear as isolated secondary maxima. In Figure 7C Cu1 and Cu2 atoms appear as bright rows with a slight asymmetry along the rows, while the $\mathrm{O}$ atoms are imaged as joint minima within the missing rows. This contrast is very similar to the simulation in the middle column in Figure $4 \mathrm{C}$ at $-1.0 \mathrm{~V}$, which gives the best match of theory and experiment. Figure 7D shows a similar contrast to $A$ but at the opposite bias polarity.

The experimental images in Figure 8 show the presence of both domains within each scan frame; in Figure $8 \mathrm{~A}, \mathrm{~B}$, and $\mathrm{D}$ the two domains appear significantly different, which can only be explained by tip asymmetry. In fact, the experimental images consistently display in one domain orientation a strong asymmetry orthogonal to the $\mathrm{O}-\mathrm{Cu}-\mathrm{O}$ rows, whereas the other domain orientation is rather symmetric in this direction with a slight asymmetry along the $\mathrm{O}-\mathrm{Cu}-\mathrm{O}$ rows. This again suggests $\mathrm{CuO}$ and $\mathrm{CuO}-$ rot tips as likely candidates. As demonstrated in Figure 8 , this is confirmed by the conclusive agreement between experiment and theory for all images and domain orientations. It is again emphasized that for each of the STM images the two domain contrasts are matched by the simulations only by rotating the tip and keeping the particular distance and bias voltage fixed. 

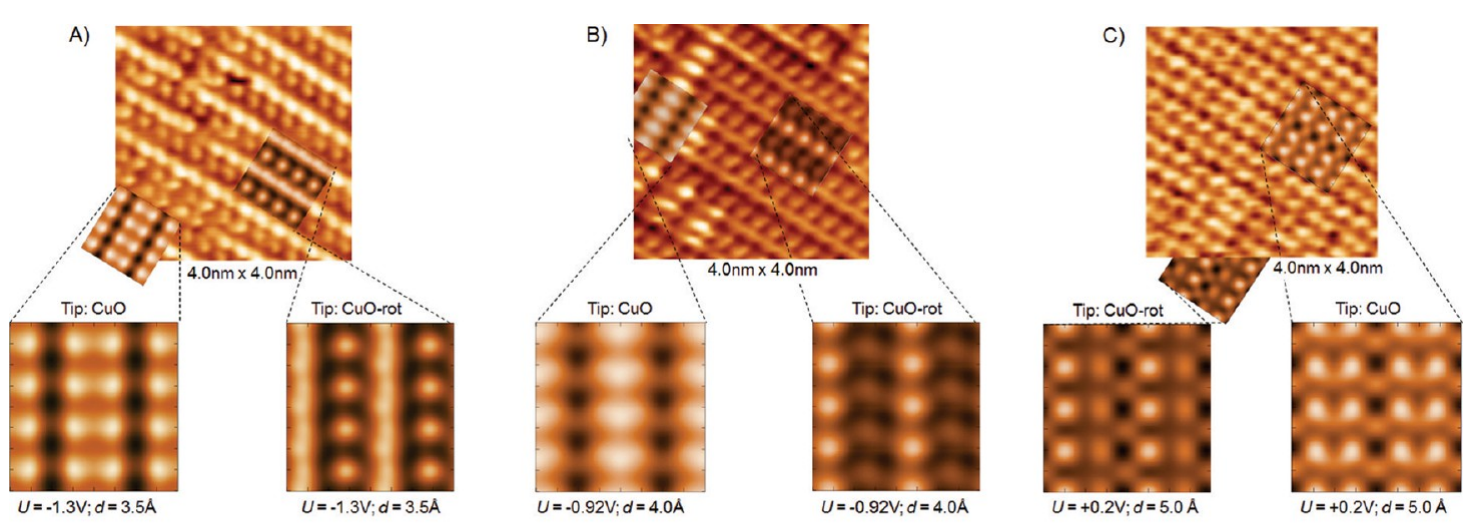

D)
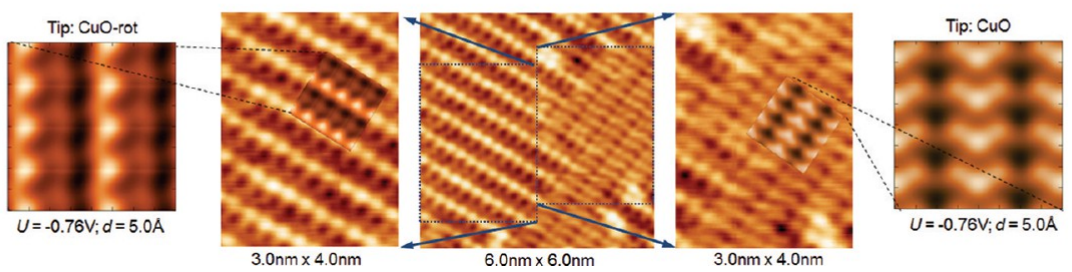

Figure 8. Comparison of experiment and theory for STM images featuring more than one domain in a single scan frame. The structural signatures of both domain orientations in each image can be reproduced if $\mathrm{CuO}$ and $\mathrm{CuO}$-rot tips are employed in the simulations. Experimental imaging parameters: $-1.30 \mathrm{~V} / 0.42 \mathrm{nA}(\mathrm{A}),-0.92 \mathrm{~V} / 0.41 \mathrm{nA}(\mathrm{B}),+0.20 \mathrm{~V} / 0.15 \mathrm{nA}$ (C), and $-0.76 \mathrm{~V} / 1.06 \mathrm{nA}(\mathrm{D})$.

\section{DISCUSSION}

Tip Stability. In our systematic comparison of experimental and simulated STM images, we found that most experimental images were acquired with oxygenterminated tips (CuO and CuO-rot). All remaining cases where tip assignments were completed indicated $\mathrm{Cu}-\mathrm{CuO}$ and $\mathrm{Cu}-\mathrm{CuO}-$ rot tip configurations as best matches. Interestingly, we did not find a single experimental image suggesting a pure Cu tip. In this context it should be noted that due to the extensive computing times required to generate a simulated STM image and the large number of experimental STM data pooled in our database, not all available experimental images could be matched with theory by targeted simulations. Therefore, it is difficult to provide accurate quantitative statistics. However, on the basis of the simulations shown in Figures 4 and 5 and the guidelines for contrast formation for the different tips (see the following section), we estimated that about $50 \%$ of our experimental images point to an oxygen-terminated tip (CuO, CuO-rot), 20\% to a side-contaminated tip ( $\mathrm{Cu}-\mathrm{CuO}, \mathrm{Cu}-\mathrm{CuO}-$ rot), and $0 \%$ to a pure copper tip. For $30 \%$ of the considered cases a clear tip assignment could not be made. This assessment suggests that tips featuring oxygen at or near their apexes are structurally more stable in the vicinity of the copper oxide surface than pure copper tips. This implies that practically unavoidable tip-surface contacts are much more likely to end in the capture of an oxygen atom or an oxide cluster from the surface than in a pure metal tip.

To gain further insight into this subject, we computed tip-surface interactions by simulating the approach and retraction of the three different tip structures with the $\mathrm{Cu}(100)-\mathrm{O}$ surface. For these simulations, the apex structures plus a third tip layer was considered. Tips featuring copper as frontmost atom were found to interact strongly with the surface upon approach, with notable structural rearrangements occurring at the $\mathrm{O}$ site. At $d=3.75 \AA$, the surface $\mathrm{O}$ atom rises to contact the $\mathrm{Cu}$ tip, while the terminating $\mathrm{Cu}$ atom of the $\mathrm{Cu}-\mathrm{CuO}$ tip detaches toward the surface, altering the apex geometry. At this tip height, tipsurface interaction forces above the surface $\mathrm{O}$ atom range from 0.9 and $0.3 \mathrm{nN}$ for the $\mathrm{Cu}$ and $\mathrm{Cu}-\mathrm{CuO}$ tips, respectively, to only $17 \mathrm{pN}$ for the $\mathrm{CuO}$ tip $^{30}{ }^{30}$ suggesting a dramatic reduction in tip reactivity with $\mathrm{O}$ termination. As a consequence, STM imaging with a $\mathrm{CuO}$ tip is possible even at close tip-sample distances, allowing a scanning regime inaccessible to the more reactive $\mathrm{Cu}$-terminated tips. Furthermore, from these observations, a picture evolves in which the deformed Cu-terminated tips appear to serve as an intermediate in tip transformations leading to the inert O-terminated structure, which is characterized by low tip-surface interactions across all surface sites.

Guidelines for Contrast Formation with Chemically Diverse Tips. In the previous sections, we established the theoretical framework for a modeling approach that reproduced most experimentally observed contrast features as a function of tip-sample distance, bias voltage, tip orientation, and apex chemistry. This allowed us to identify the tip configurations likely to be present in actual experiments. Motivated by the good agreement between theory and experiment, in this section we derive general guidelines to understand the major contrast-determining effects. 
Toward this end, we start by noting that imaging $\mathrm{Cu}$ atoms on the $\mathrm{Cu}(100)-\mathrm{O}$ surface is very common, while only a few imaging modes allow the detection of $\mathrm{O}$ atoms. This can be explained by the conduction characteristics of the tunneling junction, which is governed by both the density of states of the sample and the tip as well as the spatial extent of the orbitals dominating the tunneling process. Therefore, distinct conduction properties of the $\mathrm{Cu}$ and $\mathrm{O}$ species lead to qualitatively different responses to changes in tip height and bias voltage. The electronic states available for tunneling into or from oxygen atoms are strongly localized and due to their $\mathrm{p}$ character also directional. In contrast, tunneling via copper atoms is dominated by a combination of $s$ and $p$ orbitals, which makes their contribution to the tunneling junction more spherical while extending spatially further into vacuum. At medium or larger scanning distances, insufficient overlap between the electronic states of the surface oxygen atoms and the probe tip will produce comparatively small current contributions at all bias voltages and result in exclusive surface $\mathrm{Cu}$ atom imaging. The local density of states (LDOS) is, however, much larger on the more densely populated oxygen orbitals than on their counterparts in copper, as shown in Figure 9. Once the tip-sample distance is reduced to distances where overlap with oxygen orbitals is achieved, the high currents from oxygen sites ultimately lead to exclusive oxygen imaging. In intermediate scanning conditions where STM current contributions from $\mathrm{Cu}$ and $O$ atoms are similar, their balance can be altered by the bias voltage.

In the following, we combine the insight gained from the theoretical and experimental data discussed so far and presented in Figures 4 through 8 to classify the prevailing imaging mechanisms as a function of the following three parameters: (i) tip chemistry: metallic, O-terminated, contaminated/mixed; (ii) bias voltage: positive/negative, high ( $\geq 1 \mathrm{~V}) /$ low $(\leq 0.4 \mathrm{~V})$; (iii) tip-sample distance: far $(6 \AA)$ /midrange $(4.5 \AA) /$ close ( $3 \AA$ ) . The imaging regimes are illustrated for each tip in the parameter space spanned by bias voltage and tip-sample distance within Figure 10.

(1) Metallic tip (Cu tip): For an all-copper tip, a simple Tersoff-Hamann approximation ${ }^{18}$ is found to deliver a satisfactory description of the tunneling mechanism, with image contrasts reflecting the density of states of the sample surface that extend considerably into vacuum (Figure 9). In this case, a negative bias voltage results in imaging mainly $\mathrm{Cu} 1$ atoms, while the Cu2 sites dominate image contrast for positive bias voltages at large and medium heights (see Figure 10A). Considerable tip reactivity inhibits close-range imaging, which rules out oxygen imaging. Since the terminating copper atom with its roughly spherical electronic orbitals dominates image contrast, little or no asymmetry can be observed.

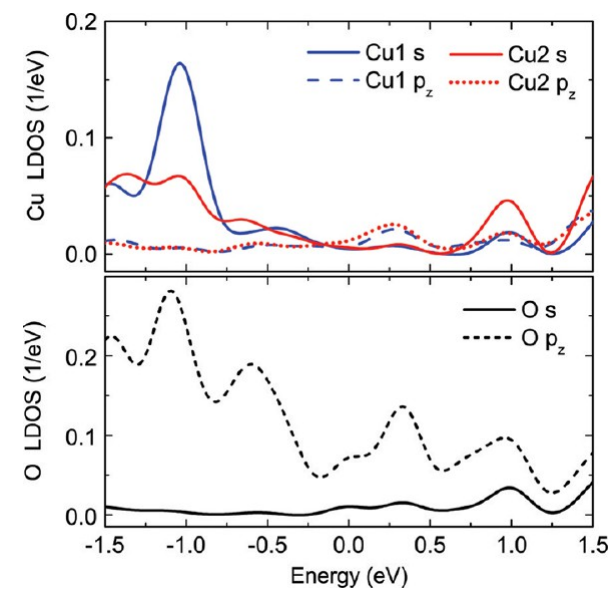

Figure 9. LDOS of $s$ and $p_{z}$ surface atom orbitals near the Fermi level, which are associated with charge flow in the $z$ direction. LDOS is displayed as number of states per $\mathrm{eV}$.
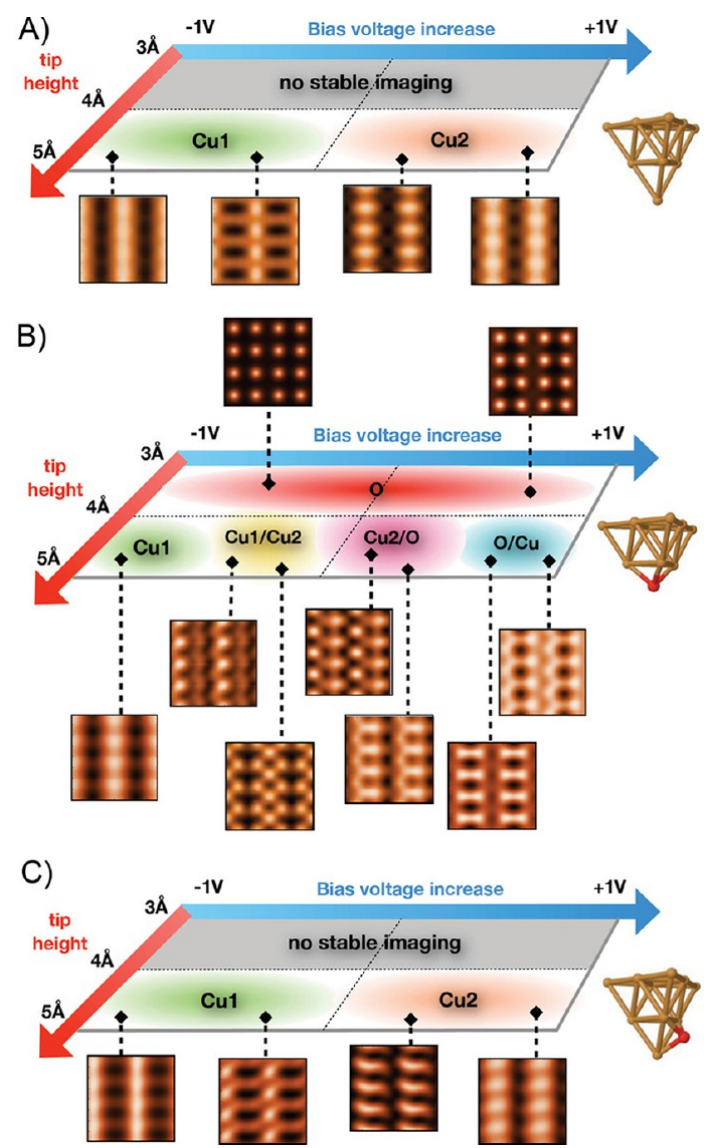

Figure 10. Imaging regimes for (A) the pure $\mathrm{Cu}$ tip, (B) the oxygen-terminated tip (CuO tip), and (C) the oxygencontaminated copper tip ( $\mathrm{Cu}-\mathrm{CuO}$ tip) in the phase space spanned by bias voltage and tip-sample distance. The labels indicate the chemical species that are preferentially imaged.

(2) Oxygen-terminated tip (CuO tip): For a tip terminated by directional oxygen orbitals the simple assumptions made in the Tersoff-Hamann approach fail, giving rise to complicated imaging behavior. The principal regimes can be summarized as follows: (i) Far 
from sample: the localized oxygen orbitals do not experience significant overlap with surface states, and imaging is dominated by the three copper atoms located in the second tip layer with a weaker signal directly below the tip apex. With increasing bias voltage, STM image maxima shift from predominantly Cu1 surface atoms to $\mathrm{Cu} 1$ and $\mathrm{Cu} 2$ atoms, followed by mixed $\mathrm{Cu} 2 / \mathrm{O}$ imaging and finally $\mathrm{O}$ imaging, as illustrated by Figure 10B. (ii) Low tip heights: localized tipapex states can overlap with the oxygen states of the sample, allowing oxygen sites to dominate image contrasts (e.g., Figure $8 \mathrm{~A}$ ) regardless of bias voltage. Since the current is dominated by the terminating $\mathrm{O}$ atom, asymmetry effects diminish and currents are high. (iii) Midrange heights: The imaging mechanisms from (i) and (ii) mix, giving rise to complicated patterns with strong asymmetry. Similar to imaging at large distances, this regime is most sensitive to bias voltage. Our findings suggest that the bias can be used to vary the contribution of the $\mathrm{Cu}$ atom tunneling current relative to the $\mathrm{O}$ one.

(3) Side-contaminated copper tip (Cu-CuO tip): Image formation is governed by mixed current collection through both the terminating $\mathrm{Cu}$ atom as well as the contaminant $\mathrm{O}$ atom with the relative contributions shifting based on the imaging parameters. Current maps in Figure $10 \mathrm{C}$ illustrate that high bias voltages amplify the contribution of the terminating copper atom and lead to Cu-tip-like imaging, while lower voltages feature convoluted image contrasts. A remarkable feature of this tip is that the location where the maximum current flows between the sample and the tip shifts away from the front $\mathrm{Cu}$ atom toward the $\mathrm{O}$ atom as the bias voltage is reduced. This may lead to image features originating from different lattice sites as well as to image distortion compared to the real geometric arrangement on the surface. Such effects are demonstrated by the ladder- and wave-like contrasts seen with the $\mathrm{Cu}-\mathrm{CuO}$ and $\mathrm{Cu}-\mathrm{CuO}-$ rot tips (Figure 6). Further complications of this picture may arise if the tip is contaminated by more than one oxygen atom.

Generalization and Perspectives. While the guidelines above are derived for an atomically flat surface oxide, we suggest they can also aid the interpretation of STM imaging modes on other metal oxide surfaces. In defining contrast categories and describing the change with bias voltage and tip-sample distance for the different tips, we provide the means to identify metal, nonmetal, and complex (contaminated) tip terminations likely found during metal oxide STM imaging as a precursor to correctly relating the contrast details to the underlying surface features. Ultimately, we propose that deliberate tip changes and functionalization with surface material would allow different contrast modes that can be categorized and identified, supplying a considerable amount of additional information about the sample and facilitating chemically selective imaging.
The strong tendency of the tip to become contaminated with surface material when imaging oxides can easily lead to oxygen termination of the apex. According to our results, this tip configuration is comparatively inert and therefore allows imaging very close to the surface, where exclusive oxygen imaging can be achieved. This imaging mode should be particularly likely when oxygen atoms are geometrically protruding, such as the bridging oxygen sites of the rutile $\mathrm{TiO}_{2}(110)-(1 \times 1)$ surface. But on this surface STM contrasts are usually dominated by the Ti $3 d$ orbitals, which are located in between the protruding oxygen rows. However, there is also a considerable number of STM images differing from these standard contrasts with distinct asymmetric features. ${ }^{19,31}$ Furthermore, simultaneous imaging of the bridging oxygen and in-plane titanium atoms at small tip-surface distances was observed. ${ }^{32}$ Our results provide a direct explanation for many of these puzzling nonstandard contrasts. Moreover, in a recent study on the rutile $\mathrm{TiO}_{2}(011)-(2 \times 1)$ surface, changes in contrast with different feedback parameters have been used in the interpretation of two different imaging modes observed at different tip-surface distances. ${ }^{33}$ The authors suggest that these two contrasts emerge from a subtle balance between the surface geometry and the different vacuum decay length of the Ti $3 d$ and $\mathrm{O} 2 \mathrm{p}$ states, which is also in line with our conclusions.

One of the key insights provided by our work is how we can deliberately exploit the imaging conditions, in particular the bias and tip height, to achieve selective imaging. Taking advantage of the bias dependence may seem problematic for bulk oxides with a low density of states and a related limited conductivity. However, commercially available scanning probe systems increasingly allow noncontact atomic force microscopy measurements while simultaneously recording tunneling currents. This enables current collection even very close to the surface of materials with low conductivity. Furthermore, the development of methods to produce high-quality oxide thin films grown on metal substrates opens the way to explore the voltagetip height phase space required to characterize the tip structure and chemistry.

Apart from bulk oxides and oxide layers on surfaces, the derived guidelines for the interpretation of STM contrast mechanisms are likely to also apply for other compound materials including chalcogenides, pnictides, and halides. For these and similar compounds, tip contamination with surface material could lead to similar contrast mechanisms, e.g., anion imaging at small tip-sample distances.

\section{CONCLUSIONS}

We systematically investigated the STM contrast mechanisms for metal oxide surfaces using the oxygen-induced surface reconstruction on $\mathrm{Cu}(100)$ as 
a model system. In a first step, we developed the computational methodology that allowed us to calculate STM image contrasts as a function of tip chemistry, bias voltage, tip-sample distance, and relative tip orientation. Three tip structures were included in the simulations: pure copper tips, oxygen-terminated tips, and copper-terminated tips with oxygen side-contamination. The resulting library of theoretical images was then matched to an extensive database of experimental STM images. The high level of agreement between experimental and simulated contrasts allowed us to identify tip structures and their orientation relative to the sample surface during data acquisition. Most frequently, tips were found to be oxygen terminated, in agreement with our simulations of the tip-surface interaction that confirm that this represents the most stable tip structure. Furthermore, these simulations confirm that metal tips are easily contaminated by surface oxygen and are therefore rather unstable in the vicinity of the surface.

Our study identifies the fundamental mechanisms underlying contrast formation in the STM imaging of metal oxides and provides comprehensive guidelines for the interpretation of STM data derived from the analysis of these mechanisms. In particular, our combined theoretical and experimental results give a conclusive picture about effects caused by tip structure, asymmetry, chemistry, and stability. Most notably it was shown that by tuning imaging parameters, different copper sites or oxygen atoms are imaged. Ultimately, precise control of these parameters may allow new strategies to be established for element-specific imaging on surfaces of complex compounds by selectively tunneling into anionic or cationic lattice sites.

\section{METHODS}

Theory. The $\mathrm{Cu}(100)-\mathrm{O}$ surface and the Cu-based STM probe tip were modeled by local-orbital DFT methods implemented in the Open $M X^{34}$ code, while tip-sample tunneling currents were computed using the NEGF formalism. A four-layer slab model featuring the $(2 \sqrt{2} \times \sqrt{2}) R 45^{\circ}$ copper oxide surface was simulated using the OpenMX code, norm-conserving pseudopotentials, $^{35}$ PBE exchange-correlation functional, ${ }^{36}$ and a single- $\zeta$ basis set with a cutoff of 6.0 au for both $\mathrm{Cu}$ and O pseudoatomic orbitals. In the $2 \times 1$ unit cell, the geometry of the top two surface layers was optimized to a force tolerance of $0.01 \mathrm{eV} / \AA ̊$ in calculations with an energy cutoff of 200 Ry and $8 \times$ $16 \times 1$ k-point mesh. Surface reconstruction and electronic structure were found to be in good agreement with previous VASP $^{37}$ benchmark calculations for this surface ${ }^{30}$ and past theoretical and experimental data. ${ }^{38-41}$

$\mathrm{Cu}(111)$ tip models were terminated in tetrahedron-shaped metallic clusters that contained both $\mathrm{Cu}$ and $\mathrm{O}$ surface species. Stable tip-apex structures were obtained by optimizing the geometry of the two lowest tip layers, which include the apex-terminating atom, the triangular Cu layer above, and, in the case of the $\mathrm{Cu}-\mathrm{CuO}$ tip, the laterally contaminating oxygen atom that is located slightly higher than the terminating $\mathrm{Cu}$ atom (see Figure 3F). The $\mathrm{Cu}$ - and $\mathrm{CuO}$-tip geometries remained qualitatively similar, although replacing a terminating $\mathrm{Cu}$ atom with an oxygen reduced the distance from the apex termination to the upper Cu layer notably from $1.7 \AA$ to $1.15 \AA$. For the $\mathrm{Cu}-\mathrm{CuO}$ tip, the $\mathrm{Cu}$ termination was preserved with the contaminating oxygen atom positioned $0.6 \AA$ above and $1.8 \AA$ laterally away from the apex atom position, with the vertical distance between the terminating $\mathrm{Cu}$ atom and the upper $\mathrm{Cu}$ layer increasing to $1.9 \AA$.

Tip electronic structure and long-range tip-surface hopping probabilities were extracted from local-orbital DFT calculations. $^{10}$ The STM probe model with the different apex structures (Figure 3) was placed above the surface, with tip-sample distance $d$ defined as the height difference between the terminating tip-apex atom and the tallest feature of the $\mathrm{Cu}(100)-\mathrm{O}$ surface. Given the Hamiltonian and density of states extracted from independent DFT calculations for the tip and surface structures, the in-house STMAD code was applied to compute tip-sample conductance and currents as described elsewhere. ${ }^{10,42}$ The STM current was integrated in $0.05 \mathrm{eV}$ bins, and only dominant current contributions from the bottom two tip layers and the uppermost surface layer were included. Tips were moved vertically to extract $I-Z$ spectroscopy curves or laterally across the surface to compute two-dimensional STM surface images.
Tip-surface interactions were explored in a previous atomic force microscopy study $^{30}$ using the VASP code. ${ }^{37}$ With $\mathrm{Cu}$ terminated tips at tip-sample distances smaller than $4 \AA$, significant structural rearrangement of the tip or surface occurred, indicating a stable STM imaging regime roughly $5 \AA$ above the surface. Structural rearrangements of the tip and multiple scattering effects are not considered in this study, as they are negligible for O-terminated tips and for $\mathrm{Cu}$-terminated tips at $d>4 \AA$.

Experiments. The STM experiments were performed using a home-built low-temperature, ultrahigh-vacuum combined scanning tunneling/atomic force microscopy system, which allows the simultaneous recording of tunneling current and frequency shift images. ${ }^{43}$ Toward that end, electrochemically etched W and PtIr tips used to collect the tunneling current are mounted at the end of a tuning fork, which is oscillated at its resonance frequency $(\sim 28 \mathrm{kHz})$ and with amplitudes below $1 \mathrm{~nm}$ when forces were to be measured. The measurements were performed at liquid and solid nitrogen temperatures (77 and $\sim 60 \mathrm{~K}$, respectively). All STM images evaluated for the present work were recorded at constant current feedback settings. Note that oscillation was enabled during data acquisition of Figures $2 B, 6 A, B, 7 A$, and $8 A, B, D$; the evaluation of the resulting frequency shift images is, however, beyond the scope of this paper.

For the preparation of the oxygen-induced surface reconstruction, a $\mathrm{Cu}(100)$ single crystal was cleaned by cycles of $\mathrm{Ar}^{+}$-sputtering and annealing. The $(2 \sqrt{2} \times \sqrt{2}) R 45^{\circ}$ missing row reconstruction was obtained by exposing the clean $\mathrm{Cu}(100)$ surface to $1000-3000 \mathrm{~L}$ of molecular oxygen at $575 \mathrm{~K}$.

Conflict of Interest: The authors declare no competing financial interest.

Acknowledgment. Financial support from the U.S. Department of Energy (Basic Energy Sciences grant no. DE-FG0206ER15834), the National Science Foundation through the Yale Materials Research Science and Engineering Center (grant nos. MRSEC DMR-1119826 and DMR-0520495) and the Materials World Network program (grant no. MWN DMR-0806893), the Spanish MINECO (projects MAT2008-02953-E, MAT2011-023627, and CSD2010-00024), and the UAM-Banco Santander Program of Collaboration with the USA is gratefully acknowledged. Access to the Magerit (CesViMa, Madrid) and MareNostrum III supercomputers (BCS, Barcelona) was provided through the Spanish Supercomputing Network (RES, Spain). H.M. acknowledges support by the Deutsche Forschungsgemeinschaft (DFG) through the transregional collaborative research center TRR 061 (project B7). 


\section{REFERENCES AND NOTES}

1. Henrich, V. E.; Cox, P. A. The Surface Science of Metal Oxides; Cambridge University Press, 1996.

2. Meyer, J.; Hamwi, S.; Kröger, M.; Kowalsky, W.; Riedl, T.; Kahn, A. Transition Metal Oxides for Organic Electronics: Energetics, Device Physics and Applications. Adv. Mater. 2012, 24, 5408-5427.

3. Maurice, V.; Despert, G.; Zanna, S.; Bacos, M.-P.; Marcus, P. Self-Assembling of Atomic Vacancies at an Oxide/Intermetallic Alloy Interface. Nat. Mater. 2004, 3, 687-691.

4. Hwang, H. Y.; Iwasa, Y.; Kawasaki, M.; Keimer, B.; Nagaosa, N.; Tokura, Y. Emergent Phenomena at Oxide Interfaces. Nat. Mater. 2012, 11, 103-113.

5. Greiner, M. T.; Helander, M. G.; Tang, W.-M.; Wang, Z.-B.; Qiu, J.; Lu, Z.-H. Universal Energy-Level Alignment of Molecules on Metal Oxides. Nat. Mater. 2012, 11, 76-81.

6. Carlisle, C. I.; King, D. A.; Bocquet, M.-L.; Cerdá, J.; Sautet, P. Imaging the Surface and the Interface Atoms of an Oxide Film on $\mathrm{Ag}\{111\}$ by Scanning Tunneling Microscopy: Experiment and Theory. Phys. Rev. Lett. 2000, 84, 38993902.

7. Papageorgiou, A. C.; Pang, C. L.; Chen, Q.; Thornton, G. LowDimensional, Reduced Phases of Ultrathin $\mathrm{TiO}_{2}$. ACS Nano 2007, 1, 409-414.

8. Knudsen, J.; Merte, L. R.; Peng, G.; Vang, R. T.; Resta, A.; Laegsgaard, E.; Andersen, J. N.; Mavrikakis, M.; Besenbacher, F.; Low-Temperature, CO Oxidation on $\mathrm{Ni}(111)$ and on a $\mathrm{Au} / \mathrm{Ni}(111)$ Surface Alloy. ACS Nano 2010, 4, 4380-4387.

9. Diebold, U.; Li, S.-C.; Schmid, M. Oxide Surface Science. Annu. Rev. Phys. Chem. 2010, 61, 129-148.

10. Blanco, J. M.; Flores, F.; Pérez, R. STM-Theory: Image Potential, Chemistry and Surface Relaxation. Prog. Surf. Sci. 2006, 81, 403-443.

11. Hagelaar, J. H. A.; Flipse, C. F. J.; Cerdá, J. I. Modeling Realistic Tip Structures: Scanning Tunneling Microscopy of NO Adsorption on Rh(111). Phys. Rev. B 2008, 78, 161405-4.

12. Chaika, A. N.; Nazin, S. S.; Semenov, V. N.; Bozhko, S. I.; Lübben, O.; Krasnikov, S. A.; Radican, K.; Shvets, I. V. Selecting the Tip Electron Orbital for Scanning Tunneling Microscopy Imaging with Sub-angström Lateral Resolution. Europhys. Lett. 2010, 92, 46003-6.

13. Garcia-Lekue, A.; Sanchez-Portal, D.; Arnau, A.; Frederiksen, T. Simulation of Inelastic Electron Tunneling Spectroscopy of Single Molecules with Functionalized Tips. Phys. Rev. B 2011, 83, 155417-10.

14. Gross, L.; Moll, N.; Mohn, F.; Curioni, A.; Meyer, G.; Hanke, F.; Persson, M. High-Resolution Molecular Orbital Imaging Using a p-Wave STM Tip. Phys. Rev. Lett. 2011, 107, 086101-4.

15. Murphy, S.; Radican, K.; Shvets, I. V.; Chaika, A. N.; Semenov, V. N.; Nazin, S. S.; Bozhko, S. I. Asymmetry Effects in Atomically Resolved STM Images of $\mathrm{Cu}(014)-\mathrm{O}$ and W(100)-O Surfaces Measured with MnNi Tips. Phys. Rev. B 2007, 76, 245423-16.

16. Paz, O.; Brihuega, I.; Gómez-Rodríguez, J. M.; Soler, J. M. Tip and Surface Determination from Experiments and Simulations of Scanning Tunneling Microscopy and Spectroscopy. Phys. Rev. Lett. 2005, 94, 056103-4.

17. Hofer, W. A.; Foster, A. S.; Shluger, A. L. Theories of Scanning Probe Microscopes at the Atomic Scale. Rev. Mod. Phys. 2003, 75, 1287-1331.

18. Tersoff, J.; Hamann, D. R. Theory of the Scanning Tunneling Microscope. Phys. Rev. B 1985, 31, 805-813.

19. Sánchez-Sánchez, C.; González, C.; Jelinek, P.; Méndez, J.; de Andres, P. L.; Martín-Gago, J. A.; López, M. F. Understanding Atomic-Resolved STM Images on $\mathrm{TiO}_{2}(110)-(1 \times 1)$ Surface by DFT Calculations. Nanotechnology 2010, 21, 405702-10.

20. Hofer, W. A.; Redinger, J. Electronic Structure of a Realistic STM Tip: The Role of Different Apex Atoms. Philos. Mag. B 1998, 78, 519-525.

21. Calleja, F.; Arnau, A.; Hinarejos, J. J.; Vázquez de Parga, A. L.; Hofer, W. A.; Echenique, P. M.; Miranda, R. Contrast Reversal and Shape Changes of Atomic Adsorbates Measured with
Scanning Tunneling Microscopy. Phys. Rev. Lett. 2004, 92, 206101-4.

22. Picone, A.; Fratesi, G.; Brambilla, A.; Sessi, P.; Donati, F.; Achilli, S.; Maini, L.; Trioni, M. I.; Casari, C. S.; Passoni, M.; et al. Atomic Corrugation in Scanning Tunneling Microscopy Images of the Fe(001)-p(1×1)O Surface. Phys. Rev. B 2010, 81, 115450-6.

23. Ondrácek, M.; Pou, P.; Rozsíval, V.; González, C.; Jelínek, P.; Pérez, R. Forces and Currents in Carbon Nanostructures: Are We Imaging Atoms? Phys. Rev. Lett. 2011, 106, 176101-4.

24. Jensen, F.; Besenbacher, F.; Laegsgaard, E.; Stensgaard, I. Dynamics of Oxygen-Induced Reconstruction of $\mathrm{Cu}(100)$ Studied by Scanning Tunneling Microscopy. Phys. Rev. B 1990, 42, 9206-9209.

25. Leibsle, F. STM Studies of Oxygen-Induced Structures and Nitrogen Coadsorption on the $\mathrm{Cu}(100)$ Surface: Evidence for a One-Dimensional Oxygen Reconstruction and Reconstructive Interactions. Surf. Sci. 1995, 337, 51-66.

26. Lampimäki, M.; Lahtonen, K.; Hirsimaki, M.; Valden, M. Nanoscale Oxidation of $\mathrm{Cu}(100)$ : Oxide Morphology and Surface Reactivity. J. Chem. Phys. 2007, 126, 034703-7.

27. Bartels, L.; Meyer, G.; Rieder, K.-H. Controlled Vertical Manipulation of Single CO Molecules with the Scanning Tunneling Microscope: A Route to Chemical Contrast. Appl. Phys. Lett. 1997, 71, 213-215.

28. Lagoute, J.; Kanisawa, K.; Fölsch, S. Manipulation and Adsorption-Site Mapping of Single Pentacene Molecules on Cu(111). Phys. Rev. B 2004, 70, 245415-6.

29. Gross, L.; Mohn, F.; Moll, N.; Liljeroth, P.; Meyer, G. The Chemical Structure of a Molecule Resolved by Atomic Force Microscopy. Science 2009, 325, 1110-1114.

30. Baykara, M. Z.; Todorović, M.; Mönig, H.; Schwendemann, T. C.; Unverdi, Ö.; Rodrigo, L.; Altman, E. I.; Pérez, R.; Schwarz, U. D. Atom-Specific Forces and Defect Identification on Surface-Oxidized Cu(100) with Combined 3D-AFM and STM Measurements. Phys. Rev. B 2013, 87, 155414-9.

31. Diebold, U.; Lehman, J.; Mahmoud, T.; Kuhn, M.; Leonardelli, G.; Hebenstreit, W.; Schmid, M.; Varga, P. Intrinsic Defects on a $\mathrm{TiO}_{2}(110)(1 \times 1)$ Surface and their Reaction with Oxygen: A Scanning Tunneling Microscopy Study. Surf. Sci. 1998, 411, 137-153.

32. Tanner, R.; Castell, M.; Briggs, G. High Resolution Scanning Tunnelling Microscopy of the Rutile $\mathrm{TiO}_{2}(110)$ Surface. Surf. Sci. 1998, 412-413, 672-681.

33. Woolcot, T.; Teobaldi, G.; Pang, C. L.; Beglitis, N. S.; Fisher, A. J.; Hofer, W. A.; Thornton, G. Scanning Tunneling Microscopy Contrast Mechanisms for $\mathrm{TiO}_{2}$. Phys. Rev. Lett. 2012, $109,156105-5$.

34. Ozaki, T.; Kino, H. Numerical Atomic Basis Orbitals from $\mathrm{H}$ to Kr. Phys. Rev. B 2004, 69, 195113-19.

35. Morrison, I.; Bylander, D. M.; Kleinman, L. Nonlocal Hermitian Norm-Conserving Vanderbilt Pseudopotential. Phys. Rev. $B$ 1993, 47, 6728-6731.

36. Perdew, J. P.; Burke, K.; Ernzerhof, M. Generalized Gradient Approximation Made Simple. Phys. Rev. Lett. 1996, 77, 3865-3868.

37. Kresse, G.; Furthmüller, J. Efficient Iterative Schemes for $A b$ Initio Total-Energy Calculations Using a Plane-Wave Basis Set. Phys. Rev. B 1996, 54, 11169-11186.

38. Bonini, N.; Kokalj, A.; Dal Corso, A.; de Gironcoli, S.; Baroni, S. Structure and Dynamics of the Missing-Row Reconstruction on $\mathrm{O} / \mathrm{Cu}(001)$ and $\mathrm{O} / \mathrm{Ag}(001)$. Surf. Sci. 2006, 600, 5074-5079.

39. Harrison, M. J.; Woodruff, D. P.; Robinson, J.; Sander, D.; Pan, W.; Kirschner, J. Adsorbate-Induced Surface Reconstruction and Surface-Stress Changes in $\mathrm{Cu}(100) / O$ : Experiment and Theory. Phys. Rev. B 2006, 74, 165402-7.

40. Kittel, M.; Polcik, M.; Terborg, R.; Hoeft, J.-T.; Baumgärtel, P.; Bradshaw, A.; Toomes, R.; Kang, J.-H.; Woodruff, D.; Pascal, M.; et al. The Structure of Oxygen on $\mathrm{Cu}(100)$ at Low and High Coverages. Surf. Sci. 2001, 470, 311-324.

41. Zeng, H.; McFarlane, R.; Mitchell, K. A LEED Crystallographic Investigation of Some Missing Row Models for the $\mathrm{Cu}(100)-(2 \sqrt{2} \times \sqrt{2}) \mathrm{R} 45^{\circ}$-O Surface Structure. Surf. Sci. Lett. 1989, 208, L7-L14. 
42. Mingo, N.; Jurczyszyn, L.; Garcia-Vidal, F. J.; Saiz-Pardo, R.; de Andres, P. L.; Flores, F.; Wu, S. Y.; More, W. Theory of the Scanning Tunneling Microscope: $\mathrm{Xe}$ on Ni and Al. Phys. Rev. B 1996, 54, 2225-2235.

43. Albers, B. J.; Liebmann, M.; Schwendemann, T. C.; Baykara, M. Z.; Heyde, M.; Salmeron, M.; Altman, E. I.; Schwarz, U. D. Combined Low-Temperature Scanning Tunneling/Atomic Force Microscope for Atomic Resolution Imaging and SiteSpecific Force Spectroscopy. Rev. Sci. Instrum. 2008, 79, 033704-9. 